PROCEEDINGS OF THE

AMERICAN MATHEMATICAL SOCIETY

Volume 137, Number 7, July 2009, Pages 2393-2402

S 0002-9939(09)09870-0

Article electronically published on February 17, 2009

\title{
$K$-EXACT GROUPS AND COARSELY EMBEDDABLE GROUPS
}

\author{
SEMAİL ÜLGEN YILDIRIM
}

(Communicated by Marius Junge)

\begin{abstract}
In this paper we introduce the notion of $K$-exact $C^{*}$-algebras, in particular $K$-exact groups. We prove that $K$-exactness is stable under direct limits and that coarsely embeddable groups are $K$-exact groups under a technical condition.
\end{abstract}

\section{INTRODUCTION}

$K$-exactness is the $K$-theoretic analogue of the structural property known as exactness. An exact discrete group $\Gamma$ is one for which the minimal tensor product with its reduced $C^{*}$-algebra $C_{r}^{*} \Gamma$ (equivalently [6], the crossed product by $\Gamma$ ) preserves short exact sequences. A $C^{*}$-algebra $A$ is $K$-exact if the six-term sequence of $K$-theory groups is exact. Moreover, a group $\Gamma$ is called $K$-exact if the minimal tensor product by $C_{r}^{*}(\Gamma)$ preserves the $K$-theoretic six-term exact sequence regardless of whether it preserves short exact sequences of $C^{*}$-algebras. $K$-exactness is very important since it is related to notions such as $K$-amenability, $K$-nuclearity, and coarse embeddability. We present the relationship to $K$-amenability, $K$-nuclearity, and coarse embeddability. We prove that $K$-exactness is stable under direct limits. Moreover, in this paper we give a proof of a theorem that shows the relationship between coarsely embeddable groups and $K$-exact groups. To be more precise, we prove the following theorem:

Theorem 1.1. If $\Gamma$ is a countable discrete group that is coarsely embeddable into a separable infinite dimensional Hilbert space $H$ and the pair $\left(C_{r}^{*}(\Gamma), C(\beta \Gamma) \rtimes_{r} \Gamma\right)$ is a KK-split pair of $C^{*}$-algebras, then $\Gamma$ is $K$-exact.

The following two definitions help to express the technical condition in the above Theorem 1.1 better.

Definition 1.1. The subalgebra $B$ of $A$ is $K K$-split if there is an element $\mathbf{j}$ in $K K(A, B)$ such that $\mathbf{i} \hat{\otimes}_{\mathbf{A}} \mathbf{j}$ is the identity in $K K(B, B)$, where $\mathbf{i} \in K K(B, A)$ is induced by the inclusion $i: B \hookrightarrow A$.

Definition 1.2. A pair of $C^{*}$-algebras $(B, A)$ is a $K K$-split pair if the $C^{*}$-algebra $B$ is a $K K$-split subalgebra of $A$.

Received by the editors October 2, 2008

2000 Mathematics Subject Classification. Primary 46L80; Secondary 19K35, 46L06.

(C)2009 American Mathematical Society 


\section{2. $K$-EXACTNESS}

In this section we recall the full and the reduced group $C^{*}$-algebras, direct limits, and $K$-exact groups and $C^{*}$-algebras. We show in Proposition 2.7 that $K$ exact groups are stable under direct limits. Hence, $K$-exact groups share the same permanence property with exact groups and coarsely embeddable groups 9 . Also, we present the relation to $K$-amenability and $K$-nuclearity.

The fundamental tool in the calculation of the $K$-theory of $C^{*}$-algebras is the fact that a short exact sequence of $C^{*}$-algebras has an associated six-term exact sequence of $K$-theory groups [2, and it is recalled in Theorem 2.1 below.

Theorem 2.1 (2], The six-term exact sequence). For every exact sequence of $C^{*}$-algebras

$$
0 \longrightarrow I \stackrel{\varphi}{\longrightarrow} B \stackrel{\psi}{\longrightarrow} B / I \longrightarrow 0,
$$

the following associated six-term sequence in $K$-theory is exact:

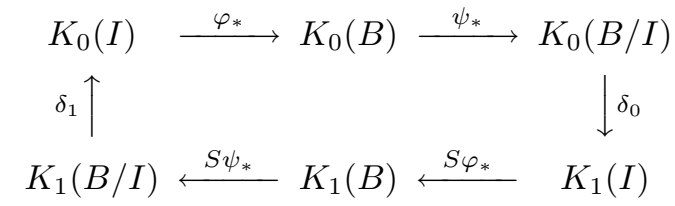

By putting a norm say $\alpha$ on the algebraic tensor product $A \odot B$ of the $C^{*}$-algebras $A$ and $B$, we make it a normed algebra, $\left(A \otimes_{\alpha} B,\|\cdot\|_{\alpha}\right)$. There is a largest and a smallest of such norms, namely the maximal and the minimal norms, $\|\cdot\|_{\max }$ and $\|.\|_{\text {min }}$, respectively. Given a $*$-homomorphism $\phi: A \rightarrow B$, and a $C^{*}$-algebra $D$, the map $\phi$ induces a map of tensor products of $C^{*}$-algebras $\phi \otimes_{\alpha} 1_{D}: A \otimes_{\alpha} D \rightarrow B \otimes_{\alpha} D$ as well as a map of $K$-theory groups $\left(\phi \otimes_{\alpha} 1_{D}\right)_{*}: K K\left(\mathbb{C}, A \otimes_{\alpha} D\right) \rightarrow K K\left(\mathbb{C}, B \otimes_{\alpha}\right.$ $D)$. The sequences of $C^{*}$-algebras (completed with respect to $\|\cdot\|_{\max }$ and $\|\cdot\|_{\text {min }}$, respectively),

$$
0 \longrightarrow I \otimes_{\max } A \stackrel{i \otimes_{\max } 1_{A}}{\longrightarrow} B \otimes_{\max } A \stackrel{j \otimes_{\max } 1_{A}}{\longrightarrow} B / I \otimes_{\max } A \longrightarrow 0
$$

and

$$
0 \longrightarrow I \otimes_{\min } A \stackrel{i \otimes_{\min } 1_{A}}{\longrightarrow} B \otimes_{\min } A \stackrel{j \otimes_{\min } 1_{A}}{\longrightarrow} B / I \otimes_{\min } A \longrightarrow 0,
$$

are not both exact. Indeed, for all $C^{*}$-algebras $B$, all ideals $I$ in $B$, and a $C^{*}$ algebra $A$, the sequence (11) is always exact [22] and implies a six-term exact sequence in $K$-theory by Theorem 2.1, whereas the sequence (2) may fail to be exact 22. The lack of exactness in the case of $\otimes_{\min }$ leads us to the definition of $K$ exactness.

Definition 2.1. A $C^{*}$-algebra $A$ is $K$-exact if for every exact sequence of $C^{*}$ algebras

$$
0 \longrightarrow I \stackrel{i}{\longrightarrow} B \stackrel{j}{\longrightarrow} B / I \longrightarrow 0,
$$

there exist $\delta$ maps $\left(\delta_{0}\right.$ and $\left.\delta_{1}\right)$ leading to an exact six-term sequence in $K$-theory of tensor product $C^{*}$-algebras:

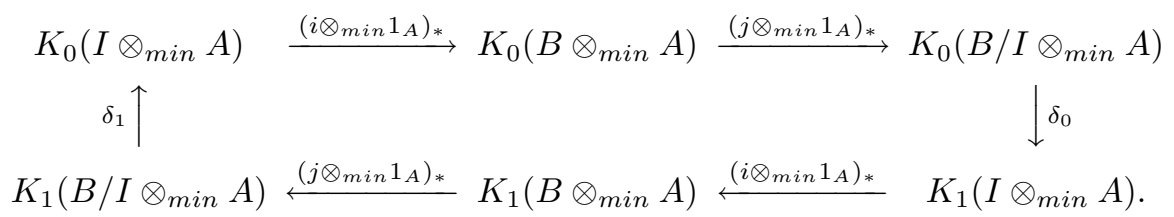


Definition 2.2. A discrete group $\Gamma$ is $K$-exact if the reduced group $C^{*}$-algebra $C_{r}^{*}(\Gamma)$ is $K$-exact.

Our examples below include certain full and reduced group $C^{*}$-algebras.

2.1. Examples. Any exact group is $K$-exact by definition of exactness. By [7, 8] and [13, Gromov's group is not exact, and it is not $K$-exact by Ozawa. Any $C^{*}$-algebra $A$ which is $K K$-equivalent to a $K$-exact $C^{*}$-algebra $B$ is $K$-exact [12]. This follows from the Kasparov product. It is defined by Cuntz that a group $\Gamma$ is $K$-amenable if and only if the full and reduced group $C^{*}$-algebras have the same $K$-theory groups [15. The next proposition states the relationship between $K$-amenable groups and the $K$-exactness of the full group $C^{*}$-algebras.

Proposition 2.2. If $\Gamma$ is $K$-amenable and $K$-exact, then $C^{*}(\Gamma)$ is $K$-exact.

Proof. $\Gamma$ is $K$-amenable implies that the $C^{*}$-algebras $C_{r}^{*}(\Gamma)$ and $C^{*}(\Gamma)$ are $K K$ equivalent 21]. For any $C^{*}$-algebra $D$, we have $C_{r}^{*}(\Gamma) \otimes_{\min } D$ and $C^{*}(\Gamma) \otimes_{\min } D$ are $K K$-equivalent, and hence they are $K$-equivalent [21]. We have the following diagram of groups:

$$
\begin{aligned}
& K_{i}\left(I \otimes_{\min } C^{*}(\Gamma)\right) \stackrel{\left(i \otimes_{\min } 1_{C^{*}(\Gamma)}\right)_{*}}{\longrightarrow} K_{i}\left(B \otimes_{\min } C^{*}(\Gamma)\right) \stackrel{\left(j \otimes_{\min } 1_{C^{*}(\Gamma)}\right)_{*}}{\longrightarrow} K_{i}\left(B / I \otimes_{\min } C^{*}(\Gamma)\right) \\
& \left(1_{I} \otimes_{\min } \lambda\right)_{*} \downarrow \quad\left(1_{B} \otimes_{\min } \lambda\right)_{*} \downarrow \quad\left(1_{B / I} \otimes_{\min } \lambda\right)_{*} \downarrow \\
& K_{i}\left(I \otimes_{\min } C_{r}^{*}(\Gamma)\right) \stackrel{\left(i \otimes_{\min } 1_{C_{r}^{*}(\Gamma)}\right)_{*}}{\longrightarrow} K_{i}\left(B \otimes_{\min } C_{r}^{*}(\Gamma)\right) \stackrel{\left(j \otimes_{\min } 1_{\left.C_{r}^{*}(\Gamma)\right)_{*}}^{\longrightarrow}\right.}{\longrightarrow} K_{i}\left(B / I \otimes_{\min } C_{r}^{*}(\Gamma)\right),
\end{aligned}
$$

and the vertical maps are isomorphisms for $i=0,1$. The exactness of the sequence on the top row follows from the exactness of the sequence on the bottom row in the diagram (5). Thus, $C^{*}(\Gamma)$ is $K$-exact.

Indeed, it is true that all $K$-amenable groups are $K$-exact [12]; hence this implies that the reduced and the full group $C^{*}$-algebras are $K$-exact.

Example 2.3. Let $\mathbb{F}_{2}$ be the free group on two generators. Since $\mathbb{F}_{2}$ is $K$-amenable [15], the full group $C^{*}$-algebra $C^{*}\left(\mathbb{F}_{2}\right)$ and the reduced group $C^{*}$-algebra $C_{r}^{*}\left(\mathbb{F}_{2}\right)$ are $K$-exact by Proposition 2.2. This also follows from the reduced group $C^{*}$ algebra $C_{r}^{*}\left(\mathbb{F}_{2}\right)$ being exact $\left[23\right.$, and hence it is $K$-exact. The full group $C^{*}$ algebra $C^{*}\left(\mathbb{F}_{2}\right)$ is $K$-exact, but it is not exact [23].

2.2. Group $C^{*}$-algebras. A unitary representation of a discrete group $\Gamma$ in a Hilbert space $H$ is a homomorphism $\pi: \Gamma \rightarrow U(H)$, where $U(H)$ is the algebra of unitary operators on $H$. The algebras derived from group $\Gamma$ are $\mathbb{C}[\Gamma], \ell^{1}(\Gamma)$, $C^{*}(\Gamma)$ and $C_{r}^{*}(\Gamma) . \mathbb{C}[\Gamma]$ is the algebra of $\Gamma$ over the complex numbers. If $a \in \mathbb{C}[\Gamma]$, then $a=\sum a_{g} u_{g}$ (finite sum with $a_{g} \in \mathbb{C}$ and basis $\left.\left(u_{g}\right)_{g}\right)$. The convolution product is given as $u_{g} \cdot u_{g^{\prime}}=u_{g \cdot g^{\prime}}$ and the involution is defined as $(c \gamma)^{*}=\bar{c} \gamma^{-1}$. The algebra $\ell^{1}(\Gamma)$ consists of elements $a=\sum a_{g} u_{g}$, where $\sum\left|a_{g}\right|<\infty$. Given a countable discrete group $\Gamma$ and a unitary representation $\pi: \Gamma \rightarrow U\left(H_{\pi}\right)$, one can extend $\pi$ to a $*$-homomorphism on $\mathbb{C}[\Gamma]$ (or $\ell^{1} \Gamma$ ) as follows: $\pi: \sum a_{g} u_{g} \rightarrow \sum a_{g} \pi(g)$. To any unitary representation $\pi$, we associate a $C^{*}$-subalgebra $C_{\pi}^{*}(\Gamma) \subseteq B\left(H_{\pi}\right)$, the closure of $\pi(\mathbb{C}[\Gamma])$ (or equivalently, $\pi\left(\ell^{1}(\Gamma)\right)$ in $B\left(H_{\pi}\right)$ ). Abstractly, one can take the completion of $\mathbb{C}[\Gamma]$ or $\ell^{1}(\Gamma)$ with respect to the norm $\|.\|_{B\left(H_{\pi}\right)}$. For example, if $t$ is the trivial representation of $\Gamma$, then $C_{t}^{*}(\Gamma) \cong \mathbb{C}$. Two group $C^{*}$-algebras derived from a group $\Gamma$ in a similar way are the full group $C^{*}$-algebra and the reduced 
group $C^{*}$-algebra $\left(C^{*}(\Gamma)\right.$ and $C_{r}^{*}(\Gamma)$, respectively). If $\lambda: \Gamma \rightarrow \mathcal{B}\left(\ell^{2}(\Gamma)\right)$ denotes the left regular representation of $\Gamma$, given by $\lambda(g)(\xi(h))=g \cdot \xi(h)=\xi\left(g^{-1} h\right)$, where $\xi \in \ell^{2}(\Gamma)$, and $g, h \in \Gamma$, then the reduced group $C^{*}$-algebra $C_{r}^{*}(\Gamma)$ is generated by the elements of $\Gamma$ acting as a unitary operator $\lambda(g)$ via the left translation on $\ell^{2}(\Gamma)$ given above. Hence, $C_{\lambda}^{*}(\Gamma) \cong C_{r}^{*}(\Gamma) \subset B\left(\ell^{2}(\Gamma)\right)$.

Definition 2.3. The reduced $C^{*}$-algebra of $\Gamma$ is defined as $C_{r}^{*}(\Gamma)=\overline{\lambda(\mathbb{C}[\Gamma])}$, where the closure is taken in the operator norm on $\mathcal{B}\left(\ell^{2}(\Gamma)\right)$.

Next, we consider a $*$-homomorphism $\alpha: H \rightarrow G$. In general, $\alpha$ will not induce a $*$-homomorphism on the reduced group $C^{*}$-algebra $C_{r}^{*}(H)$. Of course, it does induce a homomorphism $\alpha: \mathbb{C}[H] \rightarrow \mathbb{C}[G]$, but this need not be continuous with respect to the respective minimal norms. For example, $C_{r}^{*}\left(\mathbb{F}_{2}\right)$, the reduced $C^{*}$ algebra of the free group on two generators, is a simple $C^{*}$-algebra. If the trivial homomorphism $t: \mathbb{F}_{2} \rightarrow 1$ were uniformly continuous, then we would obtain a $*$ homomorphism $\hat{t}: C_{r}^{*}\left(\mathbb{F}_{2}\right) \rightarrow \mathbb{C}$ which would have to be an isomorphism. But it is easy to see that $C_{r}^{*}\left(\mathbb{F}_{2}\right)$ is not a commutative algebra. However, if $\alpha$ were injective, then a homomorphism on the reduced $C^{*}$-algebras would be induced. We will see below that this homomorphism will be injective as well.

Proposition 2.4. Let $\alpha: H \rightarrow G$ be an injective homomorphism between groups $H$ and $G$. Then there exists an injective $*$-homomorphism $\alpha_{*}: C_{r}^{*}(H) \rightarrow C_{r}^{*}(G)$.

Proof. Let $\lambda_{G}$ and $\lambda_{H}$ be the regular representations of $G$ and $H$, respectively. Since there is an injective map $\alpha$ from $H$ into $G$, one can think of representing $H$ in $\ell^{2}(G)$. We have that $\ell^{2}(G)=\ell^{2}(H \backslash G) \otimes \ell^{2}(H)$, where $H \backslash G$ is the right conjugacy class of $G$. For $a \in \mathbb{C} H, \lambda_{G}(\alpha(a))=i d_{\ell^{2}(H \backslash G)} \otimes \lambda_{H}(a)$. We have $G=\bigsqcup_{\gamma \in H \backslash G}(H$. $\gamma)$, and it follows that $\ell_{2}(G)=\bigoplus_{\gamma \in H \backslash G} \ell^{2}(H \cdot \gamma)$. We also have $\lambda_{G}(\alpha(a))=$ $\bigoplus_{\gamma \in H \backslash G} \lambda_{H}(a)$. Then $\left\|\lambda_{G}(\alpha(a))\right\|_{\ell^{2}(G)}=\left\|i d_{\ell^{2}(H \backslash G)} \otimes \lambda_{H}(a)\right\|_{\ell^{2}(H \backslash G) \otimes \ell^{2}(H)}=$ $\left\|\lambda_{H}(a)\right\|_{\ell^{2}(H)}$ as required.

Let $\mathcal{G}_{1}$ denote the category of countable groups and injective homomorphisms, and let $\mathcal{C}_{1}$ denote the category of $C^{*}$-algebras and injective $*$-homomorphisms. Then we get the following proposition.

Proposition 2.5. The reduced $C^{*}$-algebra provides a covariant functor from $\mathcal{G}_{1}$ to $\mathcal{C}_{1}$

Proof. The fact that composition and the identity homomorphism behave correctly is obvious. The only thing to check is injectivity of the induced homomorphism. This is true by Proposition 2.4 .

The full group $C^{*}$-algebras are as important as the reduced group $C^{*}$-algebras in the theory of noncommutative geometry. Next we recall the definition of the full group $C^{*}$-algebras. Let $H_{u}=\bigoplus_{\pi} H_{\pi}$ be the Hilbert sum over all isomorphism classes of representations of $\Gamma$. Then $C^{*}(\Gamma)=C_{u}^{*}(\Gamma)$ is the full $C^{*}$-algebra of $\Gamma$.

Definition 2.4. The full $C^{*}$-algebra of $\Gamma, C^{*}(\Gamma)$, is the completion of $\mathbb{C}[\Gamma]$ under the norm $\|a\|_{C^{*}(\Gamma)}=\sup _{\pi}\|\pi(a)\|$, where $\pi$ is a unitary operator and $a \in \mathbb{C}$.

The universal property implies that for all unitary representations $\pi$, there exists a unique extension of $C_{r}^{*}(\Gamma) \rightarrow B\left(H_{\pi}\right)$ to $C^{*}(\Gamma)$. For a given countable discrete group $\Gamma$, there is a quotient map $Q$ between $C^{*}(\Gamma)$ and $C_{r}^{*}(\Gamma), Q: C^{*}(\Gamma) \rightarrow C_{r}^{*}(\Gamma)$. 
This map becomes isomorphic when the group $G$ is amenable. The induced $K$ theory map $Q^{*}: \mathrm{K}\left(C^{*}(\Gamma)\right) \rightarrow \mathrm{K}\left(C_{r}^{*}(\Gamma)\right)$ is isomorphic when $\Gamma$ is $K$-amenable, 15. Note that the map $C^{*}(\Gamma) \rightarrow C_{r}^{*}(\Gamma)$ is not injective for the free group $\mathbb{F}_{2}$, since $\mathbb{F}_{2}$ is not amenable. We give some examples of $K$-exact groups and $C^{*}$-algebras next. Indeed, in the next section we see that $K$-exactness is stable under direct limits.

2.3. Direct limits and $K$-exactness. Taking direct limits of $C^{*}$-algebras or groups helps to build new ones. In particular, consider the inductive sequence of groups with injective homomorphisms $\left\{\Gamma_{i}, \mu_{i}\right\}$ with direct $\operatorname{limit} \Gamma=\lim \Gamma_{i}$. For each $\Gamma_{i}$, we have the full and the reduced group $C^{*}$-algebras. The next proposition will make it clear that the limits of these $C^{*}$-algebras are again group $C^{*}$-algebras. By the continuity property of $K$-theory, we get that $K$-exactness is stable under direct limits. If we also assume that each $\Gamma_{i}$ is $K$-exact, then we get that $C_{r}^{*}(\Gamma)$ is $K$-exact. Moreover, $C^{*}(\Gamma)$ becomes $K$-exact if each $C^{*}\left(\Gamma_{i}\right)$ is $K$-exact.

Proposition 2.6. $\lim _{\longrightarrow} C_{r}^{*}\left(\Gamma_{i}\right) \cong C_{r}^{*}(\Gamma)$ and $\lim _{\longrightarrow} C^{*}\left(\Gamma_{i}\right) \cong C^{*}(\Gamma)$ for $\left\{\Gamma_{i}, \mu_{i}\right\}$ given as above.

Proof. We prove the first equality, and the second equality follows similarly. It is easy to see that $\lim _{\longrightarrow} \mathbb{C}\left[\Gamma_{i}\right] \cong \mathbb{C}[\Gamma]$ as $*$-algebras. The norm on $\mathbb{C}[\Gamma]$ which yields $C_{r}^{*}(\Gamma)$ induces a norm on $\underset{\longrightarrow}{\longrightarrow} \mathbb{C}\left[\Gamma_{i}\right]$, and it will be sufficient to show that the completion with respect to this norm is $\lim _{\longrightarrow} C_{r}^{*}\left(\Gamma_{i}\right)$.

Let $x \in \underset{\lim }{\longrightarrow}\left[\Gamma_{i}\right]$. Then there exists an $n$ such that $x \in \mathbb{C}\left[\Gamma_{n}\right]$. Using the isomorphism $\underset{\lim }{\longrightarrow}\left[\Gamma_{i}\right] \cong \mathbb{C}[\Gamma]$, it is easy to see that the norm of $x$ is just $\|x\|_{C_{r}^{*}\left(\Gamma_{n}\right)}$. On the other hand, the inclusions

$$
\stackrel{\lim }{\longrightarrow}\left[\Gamma_{i}\right] \rightarrow \lim ^{\text {alg }} C_{r}^{*}\left(\Gamma_{i}\right) \rightarrow \underline{\lim } C_{r}^{*}\left(\Gamma_{i}\right)
$$

show that the norm induced from including into the direct limit is also $\|x\|_{C_{r}^{*}\left(\Gamma_{n}\right)}$. Thus, the norms, and hence the respective completions agree. It is direct to check that $\lim _{\longrightarrow}\left[\Gamma_{i}\right]$ is dense in $\lim _{\longrightarrow} C_{r}^{*}\left(\Gamma_{i}\right)$. This completes the proof.

Proposition 2.7. The direct limit of $K$-exact groups is $K$-exact.

Proof. Let $\lim \Gamma_{n}=\Gamma$, and assume that each $\Gamma_{n}$ is $K$-exact, for each $n$. Since the maps for groups in the direct limit are one-one, we get maps on reduced $C^{*}$ algebras. The continuity property in $K$-theory implies that $\lim _{\longrightarrow} K_{*}\left(C_{r}^{*}\left(\Gamma_{n}\right)\right)=$ $K_{*}\left(C_{r}^{*}\left(\lim \Gamma_{n}\right)\right)$. Next, we take an exact sequence of $C^{*}$-algebras $\vec{B}$ and its ideal $I$. We tensor each term $C_{r}^{*}\left(\Gamma_{n}\right)$ for every $n$. If we take the $K$-theory of each sequence of $C^{*}$-algebras (not necessarily exact), each sequence of abelian groups becomes exact by the assumption that each $\Gamma_{n}$ is $K$-exact. Next, we take the limit of the exact $K$-theory sequences. Since the direct limit of exact sequences of abelian groups is exact [20, the limiting sequence will be exact.

A similar argument yields the following two propositions.

Proposition 2.8. The direct limit of $K$-exact $C^{*}$-algebras is $K$-exact.

Proof. For a direct system of $\left(A_{n}, \varphi_{n}\right)$, with $\underline{\lim } A_{n}=A$, we assume that $A_{n}$ is $K$-exact, for each $n$. We get that $\lim _{\longrightarrow} K_{*}\left(A_{n}\right)=K_{*}\left(\underline{\lim } A_{n}\right)$ by continuity of the $K$-theory functor. Next, take an exact sequence and tensor it with each $A_{n}$. We are assuming that each $A_{n}$ is $K$-exact, so the tensored sequence has an exact $K$ theory sequence. The limit of the exact $K$-theory sequences will be exact, since 
the direct limit of exact sequences of abelian groups is exact. By continuity of $K$-theory, we get that $A=\lim _{\longrightarrow} A_{n}$ is $K$-exact.

Proposition 2.9. Let $\lim _{\longrightarrow} \Gamma_{n}=\Gamma$, and assume that $C^{*}\left(\Gamma_{n}\right)$ is $K$-exact for each $n$. Then $C^{*}(\Gamma)$ is $K$-exact.

Proof. The proof follows from Propositions 2.6 and 2.8 .

\section{CoArse Embeddability}

Coarsely embeddable groups have drawn significant attention for the last fifteen years. This is mainly because of their connections to the coarse Baum-Connes conjecture and the Novikov conjecture. Both the Baum-Connes and the coarse Baum-Connes conjectures have geometric implications, for instance, in particular, the Novikov Conjecture. Yu proved that coarsely embeddable groups satisfy the coarse Baum-Connes conjecture and therefore the Novikov conjecture [17. The results above suggest that the next setting where these conjectures have to be studied should be $K$-exact groups. In this paper we prove the relationship between $K$-exactness and coarse embeddability of groups into a Hilbert space $\mathcal{H}$. Indeed, Theorem 1.1 states that (under a technical condition) every coarsely embeddable countable discrete group $\Gamma$ is $K$-exact. The notion of coarsely embeddable metric spaces (into a separable infinite dimensional Hilbert space $\mathcal{H}$ ) was introduced by Gromov to express the idea of inclusion in the large scale geometry of groups [18. We recall that for metric spaces $\left(X, d_{X}\right)$ and $\left(Y, d_{Y}\right)$ the function $F: X \rightarrow Y$ is a coarse embedding if there exist nondecreasing functions $\rho_{ \pm}: \mathbb{R}_{+} \rightarrow \mathbb{R}_{+}$such that $\lim _{t \rightarrow \infty} \rho_{ \pm}(t)=\infty$ and

$$
\rho_{-}\left(d_{X}\left(x, x^{\prime}\right)\right) \leq d_{Y}\left(F(x), F\left(x^{\prime}\right)\right) \leq \rho_{+}\left(d_{X}\left(x, x^{\prime}\right)\right), \quad \text { for all } x, x^{\prime} \in X .
$$

Definition $3.1([18)$. $X$ is coarsely embeddable if there exists a coarse embedding $F$ of $X$ into a Hilbert space $H$.

One can view countable discrete groups as metric spaces by attaching a natural suitable metric to them. This allows us to study coarsely embeddable groups. The class of coarsely embeddable groups is provided mainly by the class of exact discrete groups. Finite groups; $\mathbb{Z} ; \mathbb{Z}^{n}$; amenable groups; $\mathbb{F}_{n} ;$ hyperbolic groups; one-relator groups [20]; every countable subgroup of the general linear group $G L(n, K), K$ a field [5], are all coarsely embeddable groups. Gromov's group is not a coarsely embeddable group in a Hilbert space [19.

3.1. $K$-nuclearity and $K$-exactness. In this section we recall the $K$-nuclear $C^{*}$ algebras due to Skandalis [16] and show that $K$-exactness and $K$-nuclearity are related. When compared to nuclearity, $K$-nuclearity is less restrictive. Every nuclear $C^{*}$-algebra is $K$-nuclear. However, a $K$-nuclear $C^{*}$-algebra may not be nuclear. Before we define $K$-nuclearity we recall Kasparov modules.

Definition 3.2 ([21]). Let $A$ and $B$ be $C^{*}$-algebras. $\mathcal{E}(A, B)$ is the set of all triples $(E, \phi, F)$, where $E$ is a countably generated Hilbert module over $B, \phi: A \rightarrow$ $\mathcal{B}(E)$ is a $*$-homomorphism, and $F \in \mathcal{B}(E)$, such that $[F, \phi(a)],\left(F^{2}-1\right) \phi(a)$, and $\left(F-F^{*}\right) \phi(a)$ are all in $\mathcal{K}(E)$ for all $a \in A$. The elements of $\mathcal{E}(A, B)$ are called Kasparov modules for $(A, B)$. 
Sometimes $\phi$ might be suppressed in the notation to regard $(E, F)$ as an $(A, B)$ bimodule. Next we recall a nuclear Kasparov bimodule which resembles the definition of nuclear $C^{*}$-algebras.

Definition 3.3 ([16]). A Kasparov $(A, B)$-module $(E, \phi, F)$ is nuclear if $\phi: A \rightarrow$ $\mathcal{B}(E)$ can be approximated in the topology of pointwise strong operator convergence by completely positive finite-rank contractions.

The $K$-nuclearity of a $C^{*}$-algebra is defined in the same spirit of J. Cuntz's $K$-theoretic amenability [15]. Indeed, $K$-nuclearity is the representability of an identity element in a $K K$-theory group by nuclear bimodules.

Definition $3.4([16])$. A $C^{*}$-algebra $A$ is $K$-nuclear if $1_{A} \in K K(A, A)$ is represented by a nuclear Kasparov $(A, A)$-bimodule $(E, F)$.

Group $C^{*}$-algebras of $K$-amenable groups, nuclear $C^{*}$-algebras and $K$-contractible $C^{*}$-algebras are $K$-nuclear [16]. Skandalis gave an example of a non- $K$-nuclear algebra [16], as recalled in the next paragraph.

Example 3.1. If $G$ is a connected simple Lie group of rank one and $\Gamma$ is an infinite discrete subgroup of $G$ having property $T$ of Kazhdan, then $C_{r}^{*}(\Gamma)$ is not $K$-nuclear [16. Hence, it is not strongly $K$-nuclear. (See below for its definition.)

Although it may sound true that a $K$-nuclear $C^{*}$-algebra is a $C^{*}$-algebra which is $K K$-equivalent to a nuclear $C^{*}$-algebra, we see that a $C^{*}$-algebra $A$ which is $K K$ equivalent to a nuclear $C^{*}$-algebra might be different from a $K$-nuclear algebra.

Definition 3.5. A $C^{*}$-algebra $A$ is strongly $K$-nuclear if $A$ is $K K$-equivalent to a nuclear $C^{*}$-algebra $B$.

Lemma 3.2. If a $C^{*}$-algebra $A$ is strongly $K$-nuclear, then it is $K$-nuclear.

Proof. Let $A$ be a strongly $K$-nuclear $C^{*}$-algebra. By definition, $A$ is $K K$-equivalent to a nuclear $C^{*}$-algebra. Nuclear $C^{*}$-algebras are $K$-nuclear [16, and $K$ nuclear $C^{*}$-algebras are closed under $K K$-equivalency. Hence we get that $A$ is $K$-nuclear.

If $A$ is a $K$-nuclear $C^{*}$-algebra, then it is not always true that it is strongly $K$-nuclear. The relation between $K$-exactness and $K$-nuclearity is stated in the next lemma.

Proposition 3.3. If a $C^{*}$-algebra $A$ is $K$-nuclear, then $A$ is $K$-exact. In particular, if a $C^{*}$-algebra $A$ is strongly $K$-nuclear, then $A$ is $K$-exact.

Proof. Let $A$ be $K$-nuclear. Then, for any $C^{*}$-algebra $D$ we have that $P_{A, D}$ : $A \otimes_{\max } D \rightarrow A \otimes_{\min } D$ is invertible in $K$-theory, by Proposition 3.5, in [16. Namely, $A \otimes_{\max } D$ and $A \otimes_{\min } D$ are $K K$-equivalent via $\mathbf{x}_{D} \in K K\left(A \otimes_{\max } D, A \otimes_{\min } D\right)$ and $\mathbf{y}_{D} \in K K\left(A \otimes_{\min } D, A \otimes_{\max } D\right)$. Indeed, $\mathbf{x}_{D}=\mathbf{P}_{A, D}$ and $\mathbf{y}_{D}=\tau_{D}(u)$, where $u \in K K_{n u c}(A, A)$ such that $\theta(u)=1_{A}$. For the definitions of $\tau_{D}, K K_{n u c}$ and $\theta$, see Skandalis's paper [16. We will not write the subscripts $D$ for $\mathbf{x}_{D}$ and $\mathbf{y}_{D}$. It is easily understood that they correspond to the related $C^{*}$-algebras in the expression. We define maps $\psi_{D, \mathbf{x}}: K_{i}\left(A \otimes_{\max } D\right) \rightarrow K_{i}\left(A \otimes_{\min } D\right)$ and $\psi_{D, \mathbf{y}}: K_{i}\left(A \otimes_{\min } D\right) \rightarrow$ $K_{i}\left(A \otimes_{\max } D\right)$ as $\psi_{D, \mathbf{x}}(\mathbf{z})=\mathbf{z} \otimes_{A \otimes_{\max } D} \mathbf{x}$ and $\psi_{D, \mathbf{y}}(\mathbf{t})=\mathbf{t} \otimes_{A \otimes_{\min } D} \mathbf{y}$, respectively. 
We have that $\psi_{D, \mathbf{x}}$ and $\psi_{D, \mathbf{y}}$ are inverses of each other. In the commutative diagram

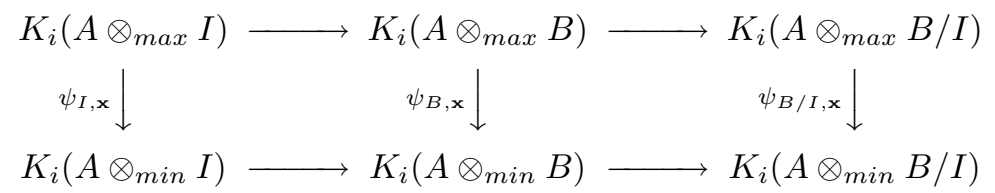

the maps $\psi$ are all isomorphisms. By the Five Lemma, the exactness of the top sequence implies the exactness of the bottom sequence. Hence, we get that $A$ is $K$-exact.

We have the following result due to Skandalis.

Proposition 3.4 ([16]). The reduced $C^{*}$-algebra $C_{r}^{*}(\Gamma)$ is $K$-nuclear if and only if $\Gamma$ is $K$-amenable .

Proposition 3.5. If a group $\Gamma$ is $K$-amenable, then $\Gamma$ is $K$-exact.

Proof. This follows from Propositions 3.3 and 3.4 .

Ozawa proved that a discrete countable group $\Gamma$ is exact if and only if the uniform Roe algebra $U C^{*}(\Gamma)$ is nuclear $\left[13\right.$. We recall that $U C^{*}(\Gamma)$ is defined as the $C^{*}$-algebra generated by $C_{r}^{*}(\Gamma)$ and $\ell^{\infty}(\Gamma)$ both acting on $\ell^{2}(\Gamma)$. The uniform Roe algebra $U C^{*}(\Gamma)$ is isomorphic to $C(\beta \Gamma) \rtimes_{r} \Gamma$ [13]. Hence, group $\Gamma$ is exact if and only if $C(\beta \Gamma) \rtimes_{r} \Gamma$ is nuclear. We prove a parallel result at the $K$-theory level. Under the assumptions that $\left(C_{r}^{*}(\Gamma), C(\beta \Gamma) \rtimes_{r} \Gamma\right)$ is a $K K$-split pair and $C(\beta \Gamma) \rtimes_{r} \Gamma$ is $K$-nuclear, we get that group $\Gamma$ is $K$-exact.

Lemma 3.6. If for a given countable discrete group $\Gamma$, the $C^{*}$-algebra $C(\beta \Gamma) \rtimes_{r} \Gamma$ is $K$-nuclear and $\left(C_{r}^{*}(\Gamma), C(\beta \Gamma) \rtimes_{r} \Gamma\right)$ is a $K K$-split pair, then $\Gamma$ is $K$-exact.

Proof. By Lemma 3.3, we get that $C(\beta \Gamma) \rtimes_{r} \Gamma$ is $K$-exact. If it is given that the pair of $C^{*}$-algebras $\left(C_{r}^{*}(\Gamma), C(\beta \Gamma) \rtimes_{r} \Gamma\right)$ is $K K$-split, then there is a $K K$-theory element $\mathbf{j}$ in $K K\left(C(\beta \Gamma) \rtimes_{r} \Gamma, C_{r}^{*}(\Gamma)\right)$ such that $\mathbf{i} \hat{\otimes}_{\mathbf{C}(\beta \Gamma) \rtimes_{\mathbf{r}} \Gamma \mathbf{j}}=1_{C_{r}^{*}(\Gamma)}$ in $K K\left(C_{r}^{*}(\Gamma), C_{r}^{*}(\Gamma)\right)$, where $\mathbf{i} \in K K\left(C_{r}^{*}(\Gamma), C(\beta \Gamma) \rtimes_{r} \Gamma\right)$ is induced by the inclusion $i: C_{r}^{*}(\Gamma) \hookrightarrow C(\beta \Gamma) \rtimes_{r}$ $\Gamma$. We let $A=C(\beta \Gamma) \rtimes_{r} \Gamma, B=C_{r}^{*}(\Gamma)$, and for any $C^{*}$-algebra $D$ the maps $\varphi_{D, \mathbf{j}}: K_{i}\left(A \otimes_{\min } D\right) \rightarrow K_{i}\left(B \otimes_{\min } D\right)$ and $\varphi_{D, \mathbf{i}}: K_{i}\left(B \otimes_{\min } D\right) \rightarrow K_{i}\left(A \otimes_{\min } D\right)$ are defined as $\varphi_{D, \mathbf{j}}()=.. \otimes_{A \otimes I} \tau_{D, \min }(\mathbf{j})$ and $\varphi_{D, \mathbf{i}}()=.. \otimes_{B \otimes I} \tau_{D, \min }(\mathbf{i})$. We consider the following commutative diagram in $K$-theory:

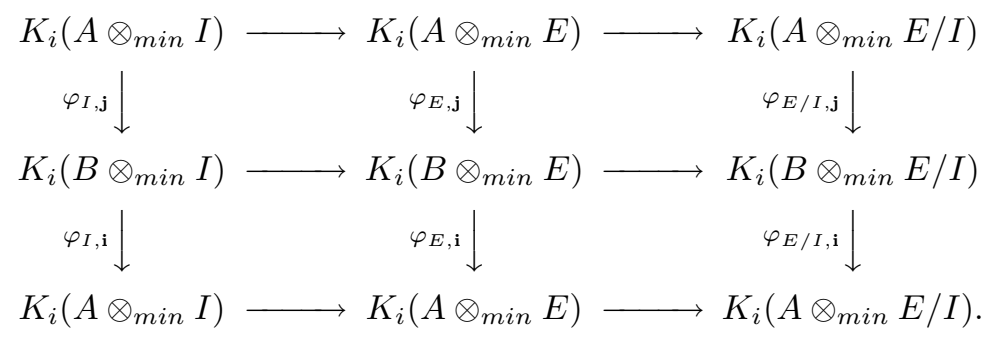

We prove that $\varphi_{D, \mathbf{j}} \circ \varphi_{D, \mathbf{i}}(z)=z, z \in B \otimes_{\min } D$ for all $C^{*}$-algebras $D$. This follows from the following equations: $\varphi_{D, \mathbf{j}} \circ \varphi_{D, \mathbf{i}}(z)=z \otimes_{B \otimes D} \tau_{D, \min }(\mathbf{i}) \otimes_{A \otimes D} \tau_{D, \min }(\mathbf{j})=$ $z \otimes_{B \otimes D} \tau_{D, \min }\left(\mathbf{i} \otimes_{A} \mathbf{j}\right)=z \otimes_{B \otimes D} \tau_{D, \min }\left(\mathbf{1}_{B}\right)=z \otimes_{B \otimes D} \mathbf{1}_{B \otimes D}=z$. We get that the groups $K_{i}\left(B \otimes_{\min } I\right), K_{i}\left(B \otimes_{\min } E\right)$, and $K_{i}\left(B \otimes_{\min } E / I\right)$ are direct summands of the groups $K_{i}\left(A \otimes_{\min } I\right), K_{i}\left(A \otimes_{\min } E\right)$, and $K_{i}\left(A \otimes_{\min } E / I\right)$, respectively. Hence, exactness of the top sequence implies exactness of the sequence in the middle of the diagram above. We get that $\Gamma$ is $K$-exact. 
In the next section we prove the main Theorem 1.1, which describes the relationship between $K$-exact and coarsely embeddable groups.

3.2. Proof of Theorem 1.1. In this section we prove the main Theorem 1.1, which states that under a technical condition coarse embeddability implies $K$-exactness. We use the fact that group $\Gamma$ being coarsely embeddable implies that $\Gamma$ is $K$-nuclear. We prove the main theorem when we combine this fact with the result above given as when $\left(C_{r}^{*}(\Gamma), C(\beta \Gamma) \rtimes_{r} \Gamma\right)$ is a $K K$-split pair of $C^{*}$-algebras and $C(\beta \Gamma) \rtimes_{r} \Gamma$ is $K$-nuclear, then $\Gamma$ is $K$-exact.

Proof. We assume that group $\Gamma$ is coarsely embeddable and $\left(C_{r}^{*}(\Gamma), C(\beta \Gamma) \rtimes_{r} \Gamma\right)$ is a $K K$-split pair of $C^{*}$-algebras. By Skandalis, Tu and $\mathrm{Yu}$, the group $\Gamma$ is coarsely embeddable if and only if the groupoid $\beta \Gamma \rtimes \Gamma$ has the Haagerup property [3, 8]. This implies that the groupoid $\beta \Gamma \rtimes \Gamma$ is $K$-amenable [11. Hence, we have that the $C^{*}$-algebras $C(\beta \Gamma) \rtimes_{r} \Gamma$ and $C(\beta \Gamma) \rtimes \Gamma$ are $K$-nuclear [10, [Proposition 4.17]. Hence, we get that $\Gamma$ is $K$-exact, by Lemma 3.6.

\section{REFERENCES}

[1] A. Connes, J. Cuntz, E. Guentner, N. Higson, J. Kaminker, J.E. Roberts, Noncommutative Geometry, Lecture Notes in Math., vol. 1831, Springer-Verlag, Berlin, 2004. MR2067646 (2005b:00021)

[2] M. Rørdam, F. Larsen, N. J. Laustsen, An Introduction to K-theory for $C^{*}$-algebras, London Math. Soc. Student Texts, 49, Cambridge Univ. Press, Cambridge, 2000, 1-239. MR1783408 (2001g:46001)

[3] N. Higson, J. Roe, Coarse Baum-Connes conjecture and groupoids, Topology, 41 (2002), $807-834$.

[4] N. Higson, J. Roe, On the coarse Baum-Connes conjecture, Novikov Conjectures, Index Theorems and Rigidity, Vol. 2 (Oberwolfach, 1993), London Math. Soc. Lecture Note Ser., 227, Cambridge Univ. Press, Cambridge, 1995, 227-254. MR1388312 (97f:58127)

[5] E. Guentner, N. Higson, S. Weinberger, The Novikov conjecture for linear groups, Publ. Math. Inst. Hautes Études Sci., 101 (2005), 243-268. MR2217050 (2007c:19007)

[6] E. Kirchberg, S. Wassermann, Permanence properties of $C^{*}$-exact groups, Doc. Math, 4 (1999), 513-558 (electronic). MR1725812(2001i:46089)

[7] E. Guentner, J. Kaminker, Exactness and uniform embeddability of discrete groups, J. Lond. Math. Soc., 70 (2004), 703-718. MR2160829 (2006i:43006)

[8] E. Guentner, J. Kaminker, Exactness and the Novikov conjecture, Topology, 41 (2002), no. 2, 411-418. MR1876896 (2003e:46097a)

[9] M. Dadarlat, E. Guentner, Constructions preserving Hilbert space uniform embeddability of discrete groups, Trans. Amer. Math. Soc., 355 (2003), 3253-3275. MR 1974686(2004E:20070)

[10] J. L. Tu, La conjecture de Baum-Connes pour les feuilletages moyennables, $K$-theory, $\mathbf{1 7}$ (1999), 215-264. MR1703305 (2000g:19004)

[11] J. L. Tu, The Baum-Connes conjecture for groupoids, $C^{*}$-algebras, Springer, Berlin, 2000, 227-242. MR1798599 (2001j:46109)

[12] S. Ülgen, K-Exactness of Group $C^{*}$-algebras, Ph.D. Thesis, Department of Mathematics, Purdue University, West Lafayette, IN, 2005.

[13] N. Ozawa, Amenable actions and exactness for discrete groups, C. R. Acad. Sci. Paris Sér. I Math., 330 (2000), no. 8, 691-695. MR.1763912 (2001g:22007)

[14] P. Nowak, Coarsely embeddable metric spaces without Property A, J. Funct. Anal., 252 (2007), 126-136. MR2357352(2008i:54026)

[15] J. Cuntz, K-theoretic amenability for discrete groups, J. Reine Angew. Math., 344 (1983), 180-195. MR716254 (86e:46064)

[16] G. Skandalis, Une notion de nucléarité en K-théorie (d'après J. Cuntz), K-theory, 1 (1998), 549-573. MR953916 (90b:46131)

[17] G. L. Yu, The coarse Baum-Connes conjecture for spaces which admit a uniform embedding into Hilbert space, Invent. Math., 139 (2000), 201-240. MR1728880 (2000j:19005) 
[18] M. L. Gromov, Asymptotic invariants of infinite groups, Geometric Group Theory (A. Niblo and M. Roller, eds.), London Math. Soc. Lecture Note Ser., 182, Cambridge University Press, Cambridge, 1993, 1-295, MR1253544 (95m:20041)

[19] M. L. Gromov, Hyberbolic groups, in Essays in Group Theory, edited by S.M. Gersten, Math. Sci. Res. Inst. Publ., 8, Springer, New York, 1987, 75-263. MR919829 (89e:20070)

[20] E. Guentner, Exactness of the one relator groups, Proc. Amer. Math. Soc., 130 (2002), 1087-1093. MR 1873783 (2002m:46084)

[21] B. Blackadar, K-theory for Operator Algebras, Cambridge Univ. Press, Cambridge, 1998. MR.1656031 (99g:46104)

[22] P. A. Fillmore, A User's Guide to Operator Algebras, John Wiley and Sons, Inc., New York, 1996. MR1385461 (97i:46094)

[23] S. Wassermann, Exact $C^{*}$-algebras and Related Topics, Lecture Notes Series, 19, Global Analysis Research Center, Seoul National University, 1994. MR.1271145 (95b:46081)

Department of Mathematics, Northwestern University, 2033 Sheridan Road, Lunt Hall 223, Evanston, Illinois 60208-2370

E-mail address: sulgen@math.northwestern.edu 